\title{
The Measurement of Thickness and Velocity of Our Galaxy
}

\author{
Jihao $\mathrm{Yu}^{1}$ \\ ${ }^{1} 19$ Lamber Drive, Princeton, NJ 08540, USA \\ Correspondence: Jihao Yu, 19 Lamber Drive, Princeton, NJ 08540, USA. E-mail: eden.yu@prismsus.org
}

Received: April 302020

Accepted: May 16, 2020

Online Published: June 30, 2020

doi:10.5539/apr.v12n3p33

URL: http://dx.doi.org/10.5539/apr.v12n3p33

\begin{abstract}
Many of the ancient astronomers have emerged and made great progress in the measuring the scale and motion of our galaxy. With rapidly developed technology, we have a more ambitious goal for putting our eyesight out of the Earth. The radius of our galaxy can be easily detected via the observation of Doppler effect. The motion of our galaxy, and the thickness of the galactic plan, however, would be relatively difficult, either because what we want to know is not stable, or because no direct evidence helps. Now with the help of radio astronomy, the most efficient tool on astronomical measuring, we are able to find out how thick our galaxy really is.

Here this paper includes the method of measuring the thickness of our galaxy via the using of radio astronomy and the Doppler effect. The whole measuring is based on an 18-meter telescope which receives only radio waves with its wavelength as $21 \mathrm{~cm} .21 \mathrm{~cm}$ is the wavelength of the radiation released while interaction between the nuclei and the electron occurs. The signal is very useful for detecting matter in space. Since diffraction is unavoidable when receiving signals on the big disk, we used the measuring of FWHM (Full width at Half Maximum) to find out the real width of the signal in the thickness measurement part when the signal is supposed to be uniformly distributed in a special range, which indicates our galactic thickness. Although we have given out a relatively precise answer at the end, unknown factors, such as the idea of precise border of our galaxy, still has a great impact on it that we could not solve. So the result, especially the thickness measuring, provides a method to measure the structure and scale of our galaxy via radio astronomy generally.
\end{abstract}

Keywords: radio astronomy; rotational curve; galactic thickness; $21 \mathrm{~cm}$ wave; Full Width as Hlaf Maximum; galactice structure; Doppler effect

\section{Introduction}

Space has long been an attractive field. Human spirit always seeks to explore the unknown and to fulfill curiosity. Our galaxy, which lies in our vision, has been a mystery and fascinated so many ancestor for thousands of years. Without precise facilities, a great number of ancient astronomers made huge progress in the measuring of it, while we, with rapidly developed technology, should have a more ambitious goal for putting our eyesight out of the Earth.

The radius of our galaxy can be easily detected via the observation of the Doppler effect. Measuring the motion of our galaxy, and the thickness of the galactic plane, however, is more difficult, given that what we want to know is not stable and no direct evidence helps. Now, with the help of radio astronomy, the most efficient tool in astronomical measuring, we are able to find out how thick our galaxy really is and how our galaxy rotates - its angular velocity as a function of radius.

Here we studied it, with the most advanced technology and hearts full of passion, to use information from radio waves on a specific frequency in deep space to detect the galactic structure and how it moves.

This paper includes two parts: the method of measuring the thickness of our galaxy via the using of radio astronomy, and the discussion of angular velocity of our galaxy as a function of radius by the Doppler effect.

Our measurements are based on an 18-meter telescope which receives only radio waves with its wavelength as $21 \mathrm{~cm} .21 \mathrm{~cm}$ is the wavelength of the radiation released while hyperfine interaction occurs, which is mainly between atoms and photons, and it is an efficient way to detect protons in space. Such detection is so important because our research is based on the behavior of matter in the galaxy. Since diffraction is unavoidable when receiving signals from the galaxy, we used the measuring of FWHM (Full width at Half Maximum) to find out the real width of the signal in the thickness measurement part of this research when the signal is supposed to be uniformly distributed in a special range, which indicates our galactic thickness. 


\section{Background Information}

\subsection{How Is $21 \mathrm{~cm}$ Radiation Generated}

There are countless numbers of protons in space, and most of them are hydrogen. Each atom of hydrogen consists of a nucleus and an electron. Magnetic moments of the nucleus and the electron are either in the same direction or opposite to each other. However, the most stable state in an atom between the electron and the nucleus is when the magnetic moment of the two particles are in opposite direction. When an atom is transferred from a less stable state to a more stable state, it emits a photon, which, relating to wave particle dualism, has a wavelength of $21 \mathrm{~cm}$. This Electronmagnetic wave-or EM wave - wave can be detected by our telescope. And the difference of power, or the intensity of the detected signal, can be recognized by the number of atoms releasing this type of EM waves. Since EM waves are hardly weakened or absorbed in outer space, and most atoms which release such an EM wave are hydrogen, the signal's intensity can be deftermined by how many atoms are being observed in the sight of our telescope.

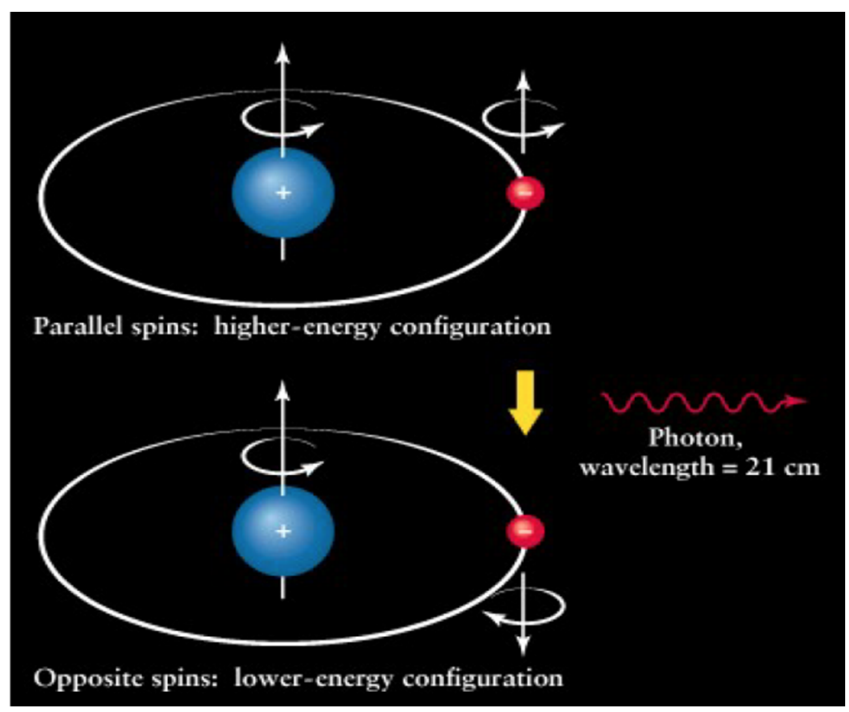

Figure $1.21 \mathrm{~cm}$ wave

\subsection{The Doppler Effect}

According to the Doppler effect, there will be little difference in the frequency of the $21 \mathrm{~cm}$ radio wave, since particles are moving at a relative speed to the Earth. Relative velocity and difference in frequency is derived through the following formula:

Here, $\mathrm{v}$ is the relative velocity at the direction of radius, if we create polar coordinates and set the observer as the original point:

$$
f=f_{0} \times \sqrt{\frac{c-v}{c+v}}^{[1]}
$$

Since $v$ is much smaller than $c$, the speed of light the formula can be written as:

$$
f=f_{0} \times(1-v / c)
$$

Here we should take $f_{0}$ as $1.4275 \times 10^{9} \mathrm{~Hz}$

By paying attention to the Doppler effect, we can identify the velocity of what we are observing, which is the rotational velocity as a function of radius. This can also help us determine the part that is nearest to the center. 


\section{Apparatus}

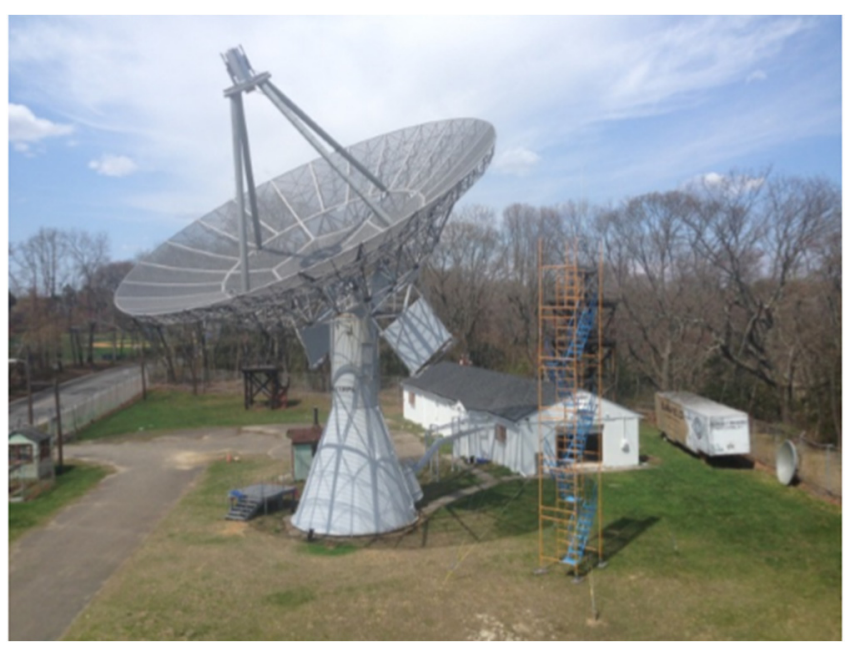

Figure 2. Our telescope

We are able to detect distant hydrogen from its release of $21 \mathrm{~cm}$ radio waves by reflecting the radio wave signals using an 18-meter telescope disk. The signal is then sent to the feed horn at the top, where it's the system analyzes its intensity. Now we move the telescope from one area of the sky to another, changing the galactic longitude and latitude that the telescope is pointing at, and thus allowing us to get a map of signal intensity among the galactic coordination.

While receiving data, the apparatus itself will do some additional processing of the data, including removing background heat noise. Later, this data, representing the signal intensity as a function of galactic longitude and latitude, is converted into a form that can be read by comput]er with the use of Universal Software Radio Peripheral (USRP).

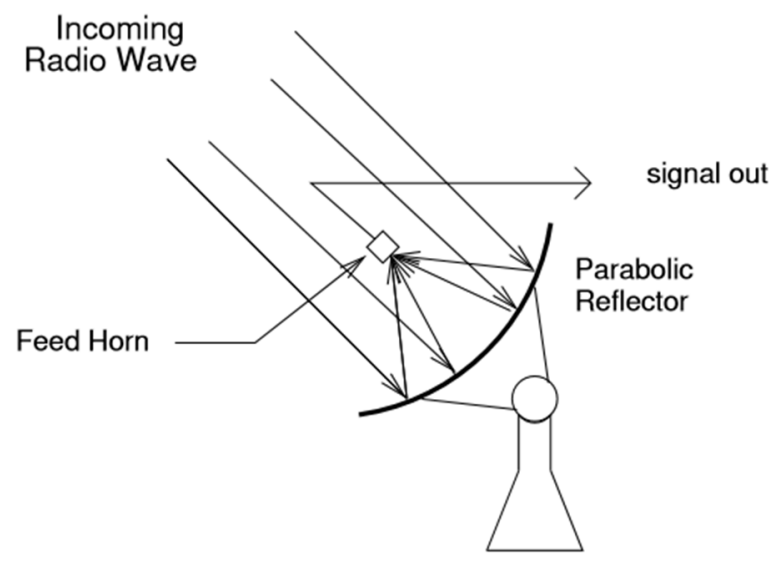

Figure 3. The telescope

\section{Analysis Method}

We use the apparatus equipment for two purposes: measuring the thickness of our galaxy, and discovering the angular velocity of our galaxy as a function of radius. Our main data analysis process was conducted in Python. Keys steps and their core ideas will be presented subsequently.

Though our project has two parts, one about thickness measurement and the other about rotational function, one part of both project requires the same method of analysis.

Since there will be some extraordinary situations in observation, like a faraway star moving at an extremely high speed that should not be considered in this measurement, which release a much higher frequency of $21 \mathrm{~cm}$ wave 
that we will consider it to be released by an extremely high-speed atom, we need to split that data and leave only the part which has the appropriate Doppler velocity. This extraordinary part of data is supposed to be released by a star in the galaxy. As our rotational speed is about $200 \mathrm{~km}$ per second, we only need the data whose velocity is between 200 and -200 kilometers per second. To do this, we use the searchsorted function:

$\mathrm{i} 1=$ np.searchsorted $(\mathrm{vDoppler}, 200)$

i2 $=$ np.searchsorted (vDoppler,-200)

In Python, we just need to split the data we received between the two edges. Assume the array we want to split is A.

$\mathrm{A}=\mathrm{A}[\mathrm{i} 1: \mathrm{i} 2]$

\subsection{Thickness Measurement}

Thickness measurement data was collected when the galactic longitude was fixed and galactic latitude was changed in a small area around zero. When the telescope is pointed in such a specific direction, signals, mainly $21 \mathrm{~cm}$ waves, will be received by the huge disk. Galactic latitude was only changed in a small area, since the galactic thickness is relatively small compared to galactic radius.

The first step is to read in files that contains the received data. We only read in the frequency as a function of latitude in order to draw the heat map. The heat map is the key step since with such a map we can figure out which part of the data we will discuss (not all the data we receive from the telescope is useful). We use equation 2 to determine the relative speed of the selected atoms, which we called "vDoppler". vDoppler here is in the form of an array.

Now we order the new version of vDoppler in the piece of the array from number i1 to number i2:

vDoppler $=$ vDoppler[i1:i2]

Since the computer figured out that il and i2 are 504 and 892 , and their difference is 388 , which is the number of elements in array vDoppler that is possible to be the relative speed of star in galaxy, we need to draw the heat map with 388 rows. In this way, we can find out which part of the data is nearest to the center.

Unfortunately, because of technical problems, our telescope provides only 13 values of galactic latitude.

Now, for a fixed longitude at 35 degrees, we read in all the power of the signal and draw a heat map as a big matrix of 13 columns and 388 rows. The X label in the heat map below refers to the relative speed, vDoppler. We assume that the velocity of our galaxy as a function of radius is a monotone function, which means that as atoms get closer to our galactic center, their relative speeds among us are higher, resulting in a more evident Doppler effect. So the point of a bigger vDoppler means it is nearest to the center of the galaxy. The Y label here refers to galactic latitude, which can be easily read by the computer. Figure 4 depicts the heat map, called Plot 1 . It shows the signal intensity of the atoms being observed in a fixed galactic longitude when galactic latitude and relative speed varies.

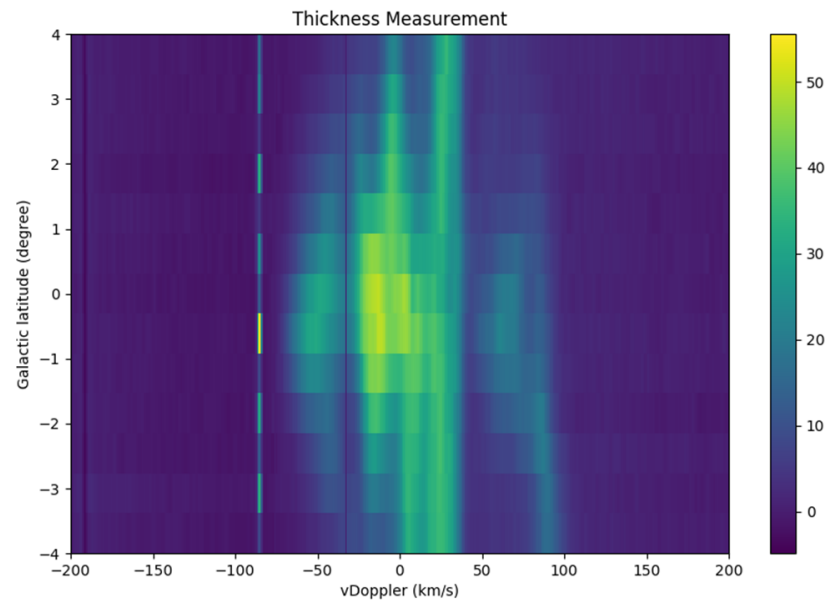

Figure 4. Doppler heat map

To explain clearly, we call a great amount of bright points located continually in the heat map a piece of 'cloud.' 
Now, there is a relatively dark line drawn in the middle of the plot. Because of the diffraction of EM waves, what we have on the left is a piece of cloud. Since the part with the biggest vDoppler lies in this cloud, that part represents the signal released from the part that is nearest to the center.

In an ideal model, what we see should not be a piece of cloud with a curved border, but rather one with a rectangular clear border. Because of unavoidable diffraction, what we have is a curve, and even the points on the bright curve line are mixed up with the nearby dark area, leading to a piece of big cloud.

Now we need to find out the curve line. In other words, we want to extract the original location of these points on the curve line, which should have the maximum power in the heat map.

To extract the maximum power in the cloud area as a function of latitude, we use a loop and the maximum function: for $y$ in range $(0,13)$ :

for $\mathrm{x}$ in range (base,base + dis +1 ):

if $\mathrm{x}==$ base + dis:

maxdata.append (max(data))

data $=[]$

else:

data.append (mapData[y][x])

while base refers to the $\mathrm{x}$ label of the left dark line and dis refers to the right dark line.

Here we have an array called maxdata as the maximum power in the cloud as a function of galactic latitude, referring to the power of $21 \mathrm{~cm}$ waves as a function of galactic latitude. Since we consider the galaxy to be a disk, on the upside and downside border the power should have a sudden decline to nearly zero. To figure out the galaxy's thickness, we just need to detect its borders' galactic latitude.

Next, we draw a plot of the maxdata as a function of galactic latitude called plot 2.

plt.plot (maxdata,np.linspace $(-4,4,13)$ )

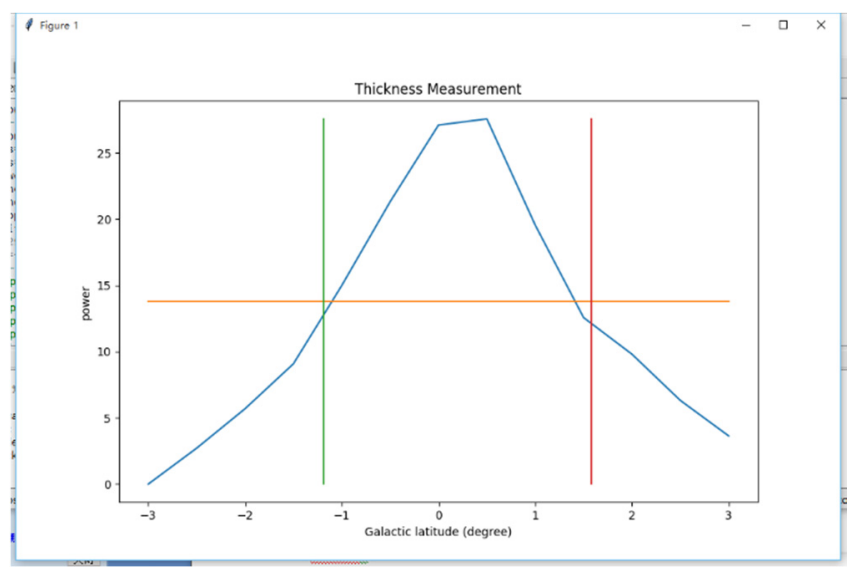

Figure 5. Maxdata plot

To get a more precise result, we will draw a Gaussian function best fit to the line chart. Here is its function:

$$
A \times e^{-\frac{\left(x-x_{0}\right)^{2}}{\text { slope }}}-h
$$

slope $=3$

$x_{0}=0.2$

$\mathrm{A}=29$

$\mathrm{h} 0=-1.5$ 


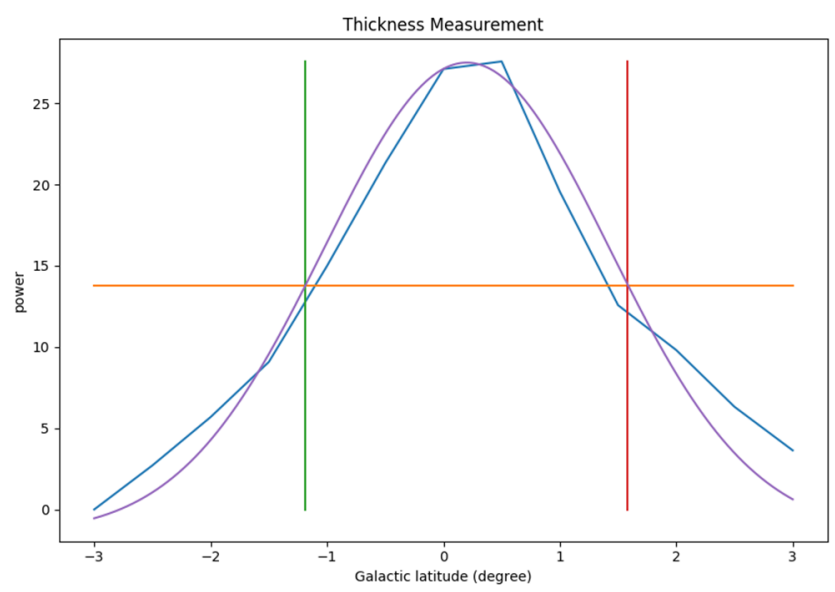

Figure 6. The fitting of HWFM

The blue line represents the raw data, and the purple line represents the fitted curve. Finally, to use the method of Full Width at Half Maximum, we draw two lines that go through the points which reach the half maximum power (the red and green lines above). By measuring the distance between two lines, we can find out the real thickness of our galaxy. The reason why FWHM works will be discussed later in theory.

\subsection{Rotational Function}

Data for this rotational function part is collected when the galactic latitude is fixed and galactic longitude varies in a large range by our telescope, the same way as in part 1 When the telescope is pointed in such a specific direction, signals, mainly $21 \mathrm{~cm}$ waves, will be received by the huge disk. For this part, galactic latitude is fixed at 0 to make sure we receive as much signal as we can. Otherwise, our data will not be so convincing. Also, our galactic longitude changes from 0 to 90 , ensuring that we will determine all the rotational velocity at different radii (the reasons will be discussed later).

Now, we need to draw a heat map for the signal intensity. The $x$ Label refers to vDoppler, and the y label to galactic longitude. Problems, like special background noise, and other things difficult to explain will be shown clearly here.

After reading in the data (frequency as a function of relative longitude), some correction is needed.

First, we change the frequency received in vDoppler. Both are in the format of arrays. Then, we extract the possible data. Only those with a vDoppler in a reasonable range are what we want. We can do this by:

vDoppler $=$ vDoppler[i1:i2]

After reading in the data (frequency as a function of relative longitude), some correction is needed

Second, for each longitude, after reading in power as a function of longitude and frequency, we extract and split the power in a loop:

power $=$ power[i1:i2]

Third, gain correction.

Furthermore, we need to eliminate the background noise. Actually, this is not the general background noise, including that 4 Kelvin one, the one of background radiation [1], and noise made in Earth naturally. We need to do this simply because it will be so unclear to see, since the original signal in all directions contains the same background signal from outer space, which is the same no matter the galactic longitude. To eliminate this useless signal, we need to find a place where no useful signal lies. Now let's look at this original map: 


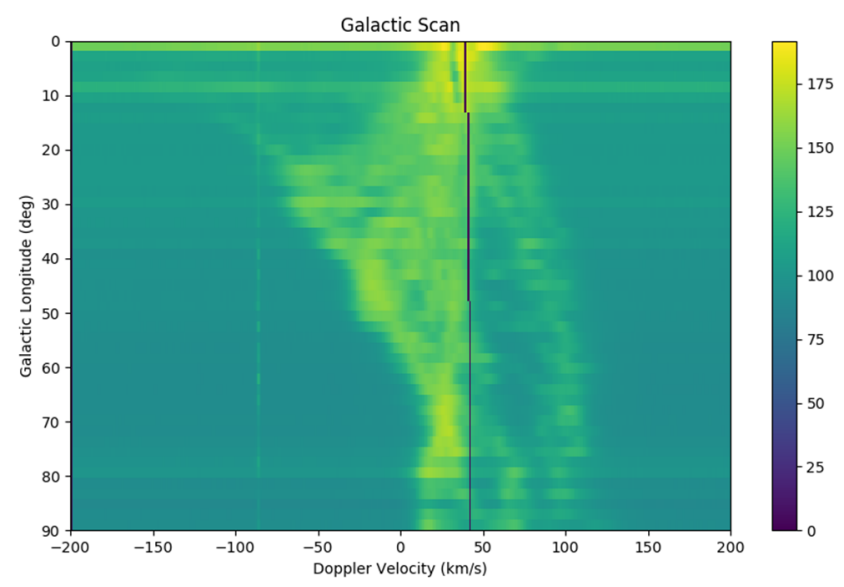

Figure 7. Doppler shift Heat map on galactic longitude

Apparently, this is an unclear map. We notice that on the left or right border of the map the signal power all remain the same, which is the lowest intensity on the whole map. Now, we change all the power values to the result of their original values minus those on the left border. We call the power on the left border as the background power, b. We write these in a loop:

power $-=\mathrm{b}$

Now the map is much better

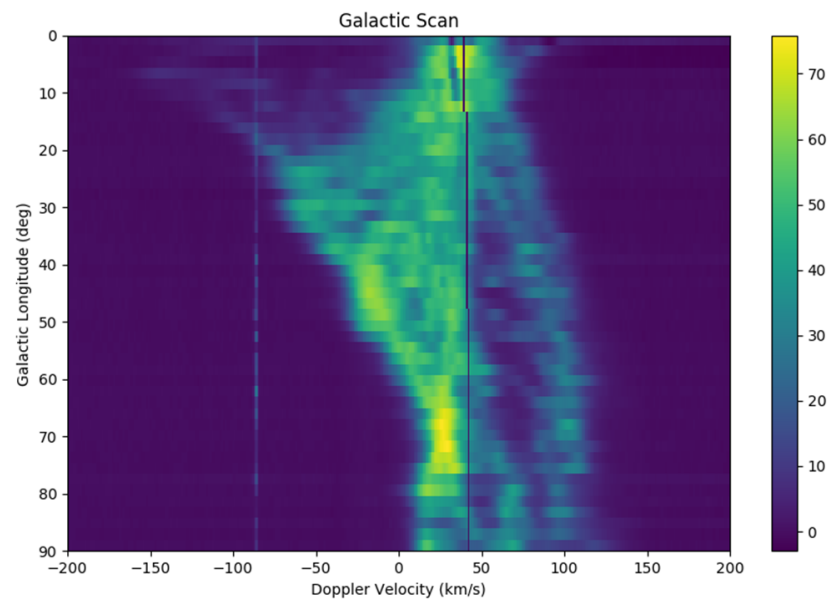

Figure 8. Corrected Doppler shift Heat map on galactic longitude

Finally, we just need to find out the Doppler Velocity value at which power greatly increases, which indicates that there is a signal that is not made of background noise.

To find out its index, we use the searchsorted function. Here we suppose the power is not significantly increasing unless it reaches 5 .

iPrime $=\min ($ np.searchsorted(power,5.),len(power)-1)

iPrime is the index of the left border of the cloud.

Its relative vDoppler can be figured out by:

$\mathrm{v}$ Prime $=$ vDoppler[iPrime $]$

But this relative velocity is only the relative velocity, which is not what we want as rotational velocity.

We do a little transform here.

We suppose vDot is Earth's velocity, figured out by another pip in Python called galcoords using the function 
called velocityprojection. vDot=alCoords.velocityProjection(fileTime,'G',gLon, 0.)

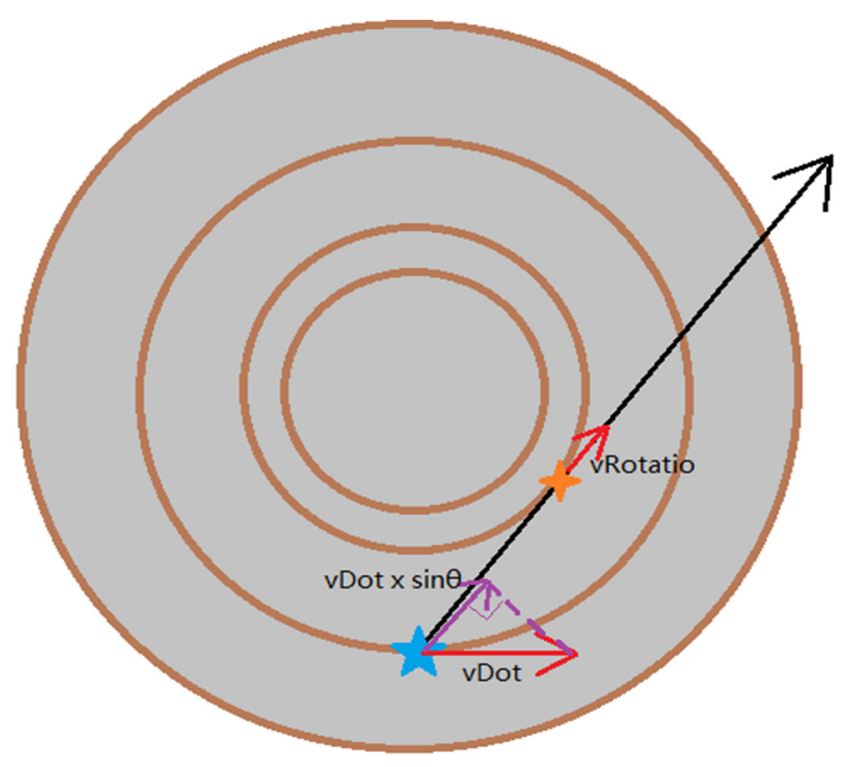

Figure 9. Velocity of the galaxy

vRotation $=\mathrm{vDot} \times \sin b-\mathrm{vDoppler}$

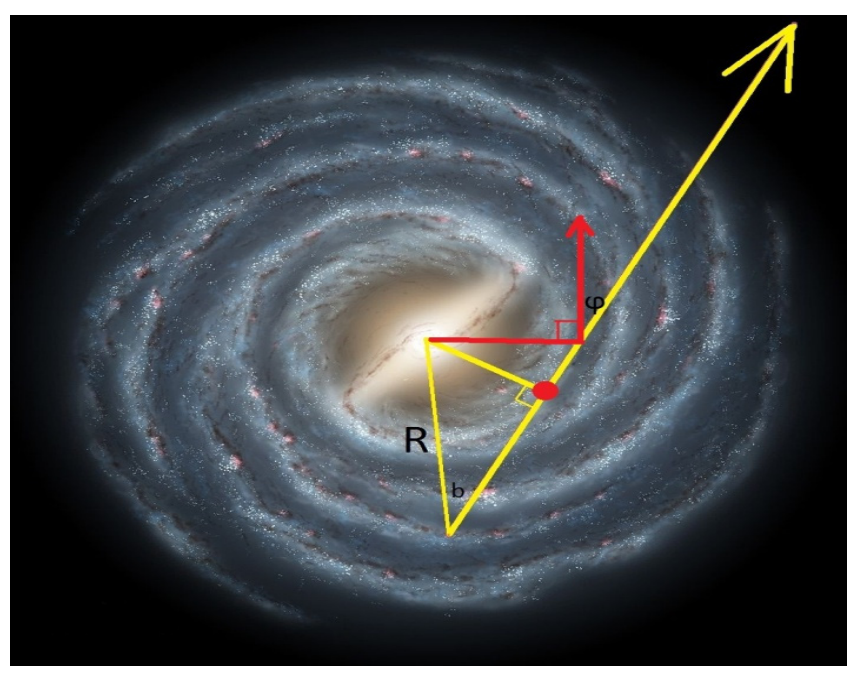

Figure 10. Velocity direction

The last step is to draw the map.

plt.plot(radius,vRotation)

\section{Result}

The result of the whole project is figured out here.

\section{Part 1}

This figure shows a conceptual model of half of our galaxy (the other half is symmetrical) and how differences in galactic latitude helps figure out the thickness. 


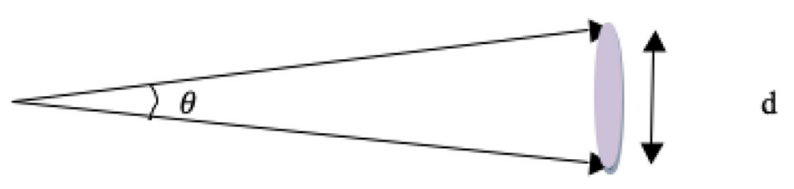

Figure 11. Thickness in our sight

$$
d=R_{0} \times \cos l \times \theta
$$

$\theta$ here is the difference in galactic latitude, which can be measured by FWHM of plot 2. According to plot 2, $\theta$ is 2.77 degree here, or 0.048346 in radians

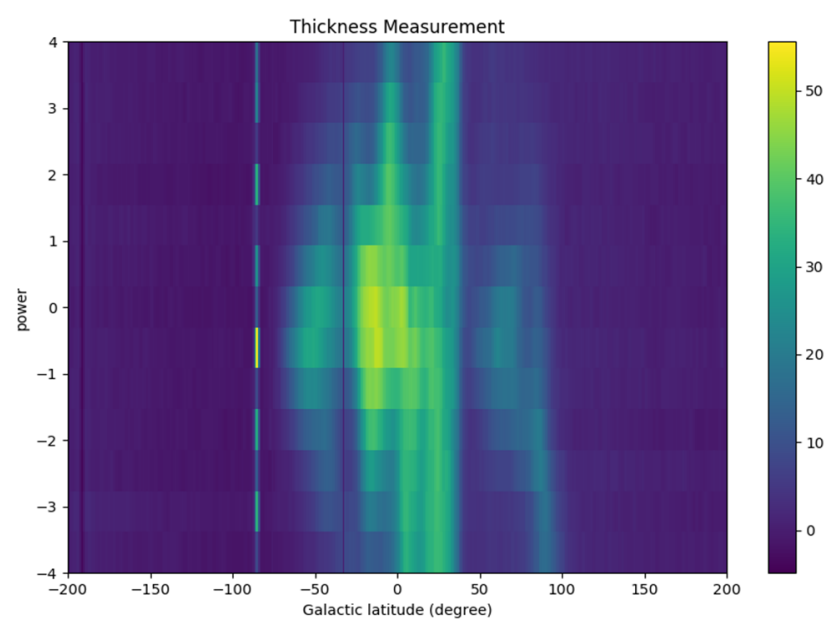

Figure 12. Borders on the heat map

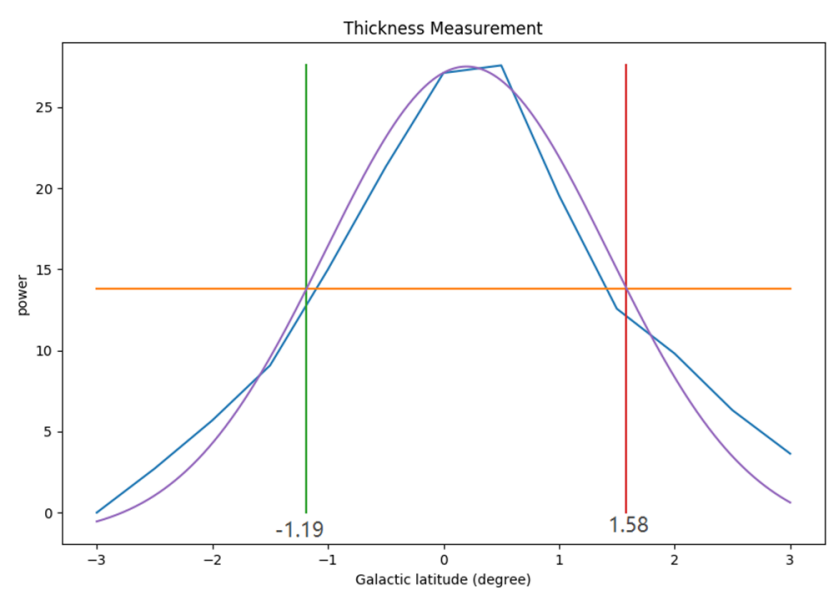

Figure 13. Result 1

$\cos 1=\cos 35=0.81915$

$\mathrm{d}=0.81915 \times 0.048346 \times 26000=1029.9$ light years.

Part 2

Here is the rotational curve. 


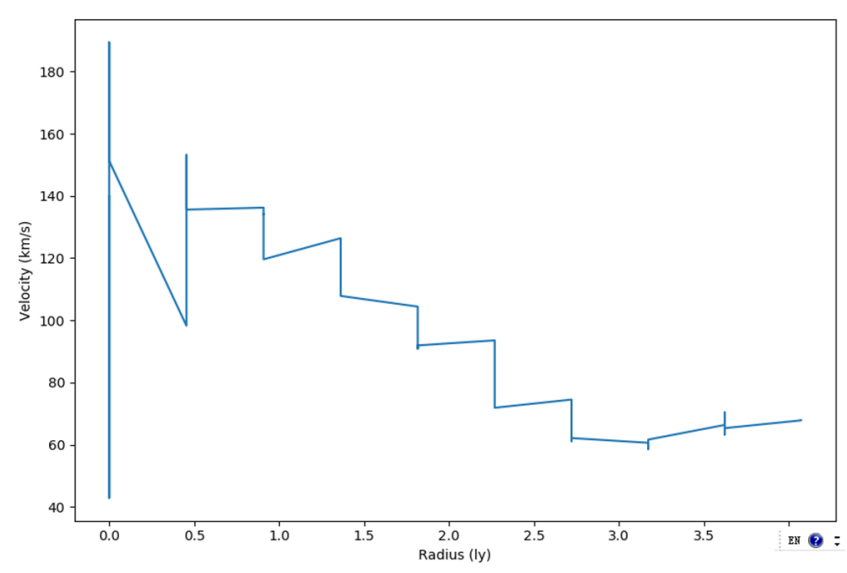

Figure 14. Result 2

The velocity is declining, but the form of the curve is still unknown. Three possible hypotheses will be discussed later.

\section{Theory}

\subsection{FWHM}

When data is collected by the 18 meter telescope, signals will be diffracted because of their wave particle dualism. In this way, the angular resolution of our telescope (the minimum angular distance that the telescope can detect) is:

$$
\Delta \theta=1.22 \times \frac{\lambda}{D}
$$

here $\mathrm{D}$ is the diameter of our telescope's disk, and $\lambda$ is the wavelength of $21 \mathrm{~cm}$ radio wave, which is, as it reads, $21 \mathrm{~cm}$. here we have $\Delta \theta$ as about 0.8 degree.

Unfortunately, 0.8 degree, compared to the object we are observing, is big enough to produce visible diffraction, which causes a negative impact on measuring. Here we use the method of full width of half maximum, known as FWHM. In a signal being diffracted, the whole plot will be shaped as a Gaussian function, instead of a peak or a rectangle.
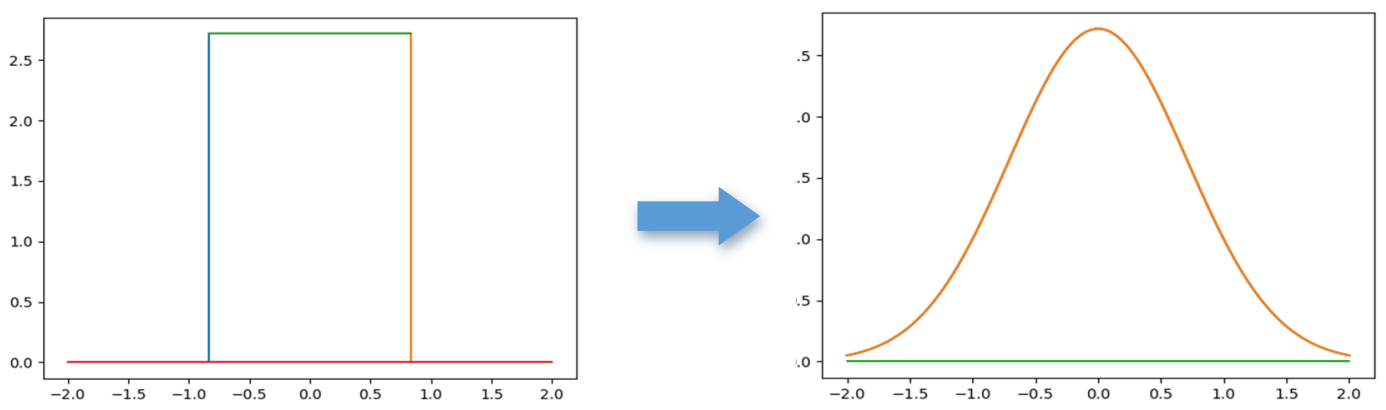

Figures 15\&16. FWHM

However, according to the conservation of energy, diffraction will not change the whole power received. In other words, the area of the plot under the curve will not be changed.

Since the Gaussian function is merely a triangle, we assume the area under such function can be expressed as:

$$
\mathrm{S}=\mathrm{h} \times \mathrm{FWHM}
$$

$h$ here is the full height, or the maximum power. FWHM is the width as half maximum. Now we can assume that the power is transferred from the upside (blue) to the downside (yellow). 


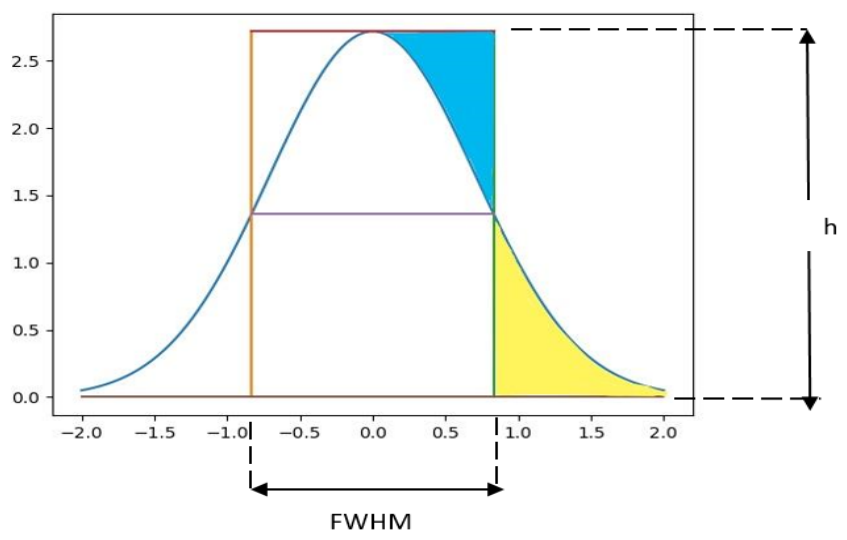

Figure 17. The prove of FWHM

In this perspective, FWHM will not change, no matter how seriously diffraction influences the shape of the signal. 6.2 To Ensure the Area We Analyze Is Nearest to the Galactic Center
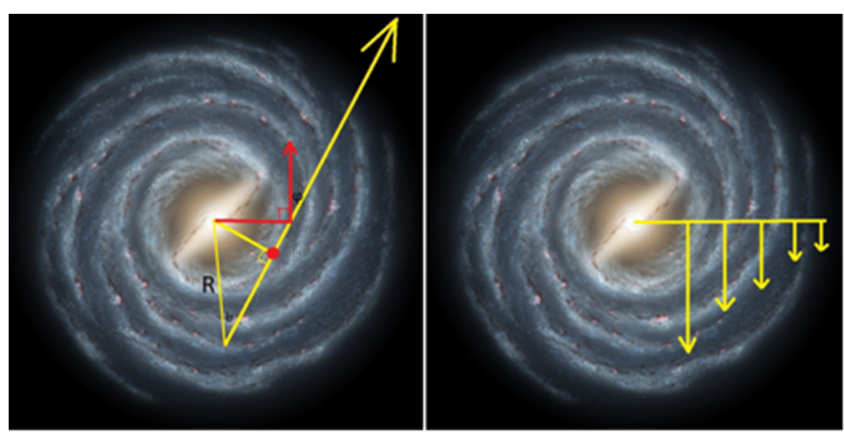

Figures 18\&19. Identify the border

Now, since the part that is nearest to the center has the minimum radius, it also has the maximum velocity according to the previous assumption. In each direction, there is only one point which is nearest to the center. Here the minimum radius point is highlighted in the graph.

Ensuring the fixed point, how are we going to find the area in the heat map represents the point nearest to center? First, we consider how the inner area rotates faster (here, faster means at a higher velocity, not only angular velocity) than the outside. Also, the most inner part's velocity is perpendicular to our observation line. vDoppler can be calculated as:

\section{vDoppler $=$ vRotation $\times \cos \varphi$ \\ vDoppler $=$ vRotation $\times \cos \varphi$}

Ensuring the fixed point, how are we going to find the area in the heat map represents the point nearest to center? First, we consider how the inner area rotates faster (here, faster means at a higher velocity, not only angular velocity) than the outside. Also, the most inner part's velocity is perpendicular to our observation line. vDoppler can be calculated as:

vDoppler $=$ vRotation $\times \cos \varphi$

vDoppler $=$ vRotation $\times \cos \varphi$

$\varphi$ here is the angle between our observation line, and the vector of speed of the atom we are observing at.

As we can see, when $\varphi$ is 90 degrees, vDoppler is largest.

Now, we realize that in this part, this most inner part we can see in our telescope, has the most significant Doppler effect, which is drawn on the leftmost part of the heat map.

For part 1, the thickness measurement, we have a heat map like this: 


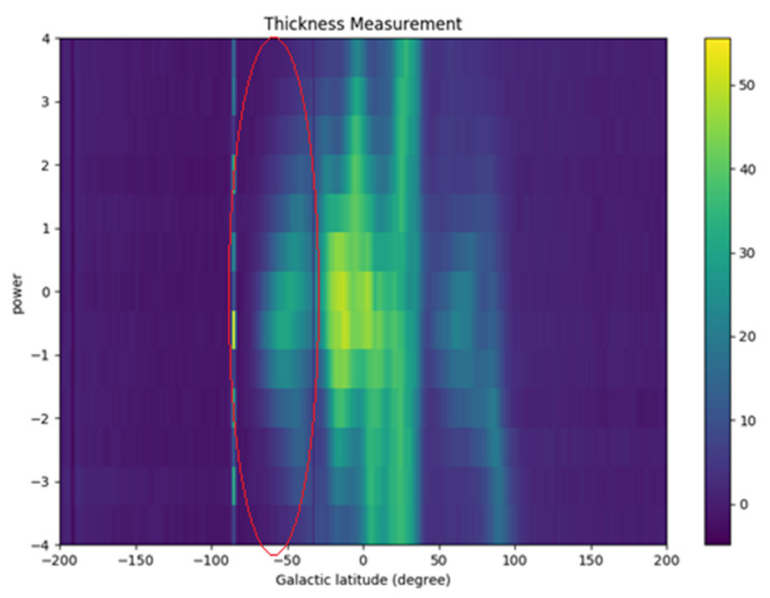

Figure 20. Other part in the heat map

Other parts of the map, which look quite similar with tribes of cloud, likely occur because of the other three arms of our galaxy. Other parts on those arms have a relative small speed, or vDoppler, making them nearer to the center of the map. On the other hand, the clouds on the other part of the map do look like tribes, making it more possible that they are formed by the arms (in the shapes of tribes). Since our measuring does include much unavoidable inaccuracy, what we have in this cloud is not a clearly drawn curve, let alone our ideal rectangle. Now, we extract the maximum point and figure out their galactic latitude. Finally, with the previously mentioned FWHM, we get the thickness.

In part two, we have a heat map like this:
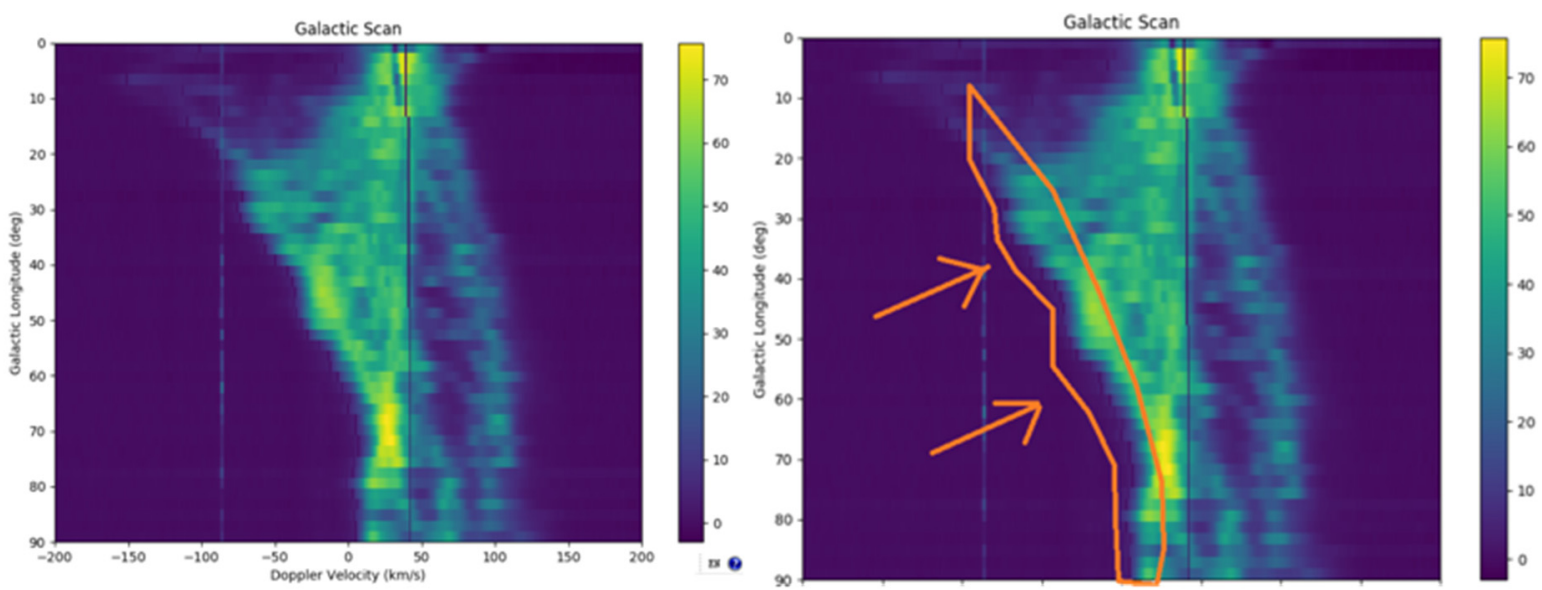

Figures $21 \& 22$. The Border of the heat map

In this map, the area in the orange circle is the part with the biggest Doppler velocity, meaning it is nearest to the center. Therefore, we can figure out its radius. Other places, similar to the thickness measurement, is probably because of the other tribes in our galaxy. 3. Models of our galaxy (Part 2)

There are three models of our galaxy's mass as a function of radius.

The Keplerian model.

According to the Keplerian model, we assume that nearly all mass zconcentrates at the center of our galaxy. This model assumes that our huge galaxy behaves like a solar system, which consists of a big-mass 'star' and other 'planets.' According to Newton's laws of motion, the rotational velocity as a function of radius is:

$$
F_{\text {inward }}=\frac{G \times M_{g} \times m}{r^{2}}
$$




$$
\begin{gathered}
F_{\text {inward }}=m \times \frac{v^{2}}{r} \\
\rightarrow v=\sqrt{\frac{G \times M_{g}}{r}} \rightarrow v=C \times r^{-\frac{1}{2}}
\end{gathered}
$$

Therefore, the plot of velocity as a function of radius should look like this (the yellow curve):

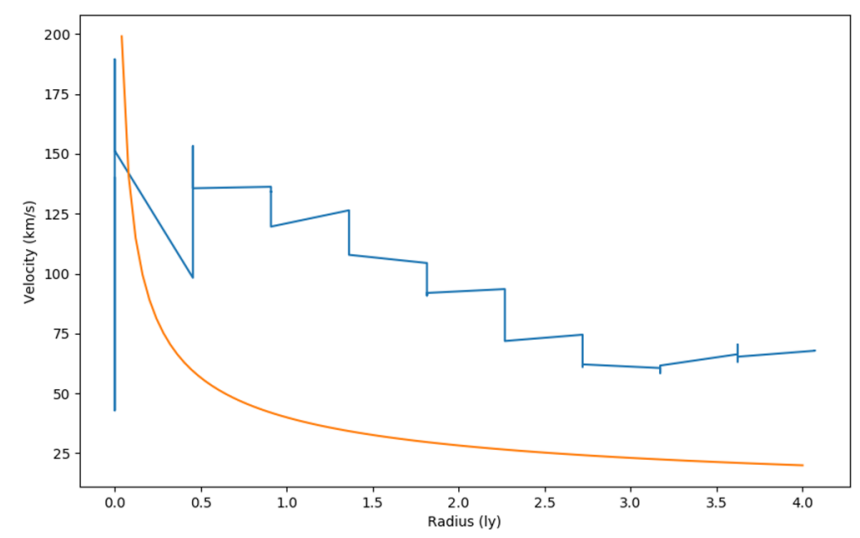

Figure 23. The fit of velocity

This is apparently not a good simulation of our galaxy. At the beginning, this model has its velocity declining too fast. This is because we have assumed nearly all the mass is located in the center. However, there is always some mass distributed on anywhere of the plane of our galaxy. 2. The Exponential Model

After former astronomers have carefully observed the light intensity on every unit area located in different outer galaxies, they found the following light intensity distribution:

$$
I=I_{0} e^{-R / R_{D}}
$$

While $I_{0}$ is a constant for each galaxy, $R_{D}$ is a special distant for each galaxy, called characteristic distance. $\mathrm{R}$ here is variable radius.

If each piece of mass emits the same amount of photon, the mass distribution will be similar:

$$
\mu=\mu_{0} e^{-R / R_{D}}
$$

$\mu$ here is the dense of the galaxy.

Now, we need to figure out the velocity of a function of radius in this model. In this term, the velocity as a function of radius is needed to be calculated carefully.

$$
m \times \frac{v^{2}}{r}=\int_{0}^{R_{0}} \int_{0}^{2 \pi} \frac{G \times m \times \mu(R) \times R}{R^{2}+R_{0}^{2}-2 \times R \times R_{0} \times \cos \theta} \times \sqrt{1-\left(\frac{R}{R_{0}} \times \cos \theta\right)^{2}} d \theta d R
$$

\section{Summary}

To get a better result in the future, and to find out problems in this measurement, we compare our results to professional measuring.

For part 1, mainly about several thousand light years. Our result is relatively small compared to most of the existing measurements, which are about several thousand light years.

One possible explanation is that our galaxy is not uniform enough. It is probably, and unfortunately, because our galaxy is so variable in its thickness as a function of longitude and radius that few of us can really give an exact answer.

A more likely explanation is that, with the using of FWHM, we only consider our galaxy as really a rigid disk, which should have had a distinct border of its thickness. The truth is, it is impossible to find a surface, whether curved or not, on which one side is our galaxy filled with countless star, and the other contains nothing but empty 
space. This means that the galaxy does not have a distinct border, but an unclear border where the density of stars is declining relatively rapidly. Our former hypothesis, which suggested that the power vs. latitude graph should be a rectangle, is not true. Instead, it should be something more like a trapezoid, since the power should be declining little by little near the galactic border. Though this led our measurement to an inaccurate result, there is nothing we can do to solve it, since no one knows the exact galactic density as a function of latitude.

For part 2, part of the mystery is still unsolved.

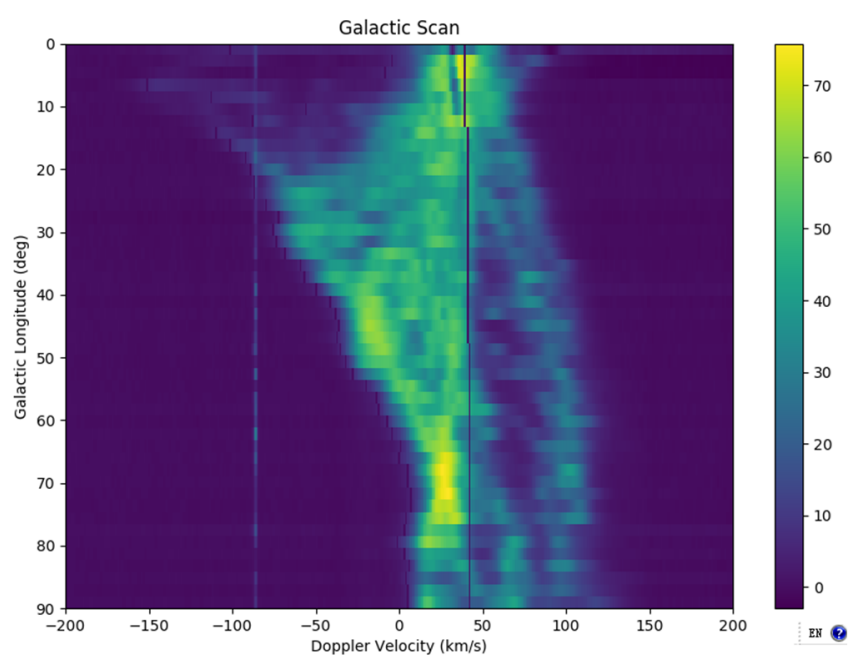

Figure 24. The intensity of the border

In this map, though we searched for the border of the cloud with intensity of 20 , we are still not sure whether this power '20' is accurate or not. On the top left part of the cloud, there is a small branch that is so dim that the computer did not consider it to be part of the cloud's border. This part is unclear compared to the border below. There is a bright but unclear border, recognized by the computer on the right. We may start to wonder which side, the left one with its dim power, or the right one with its unclear border, should be considered as a part of the real function. But if this is the truth, there should be another branch on the other side of the plot, the upper right side.

One explanation lies in the very structure of the galactic center. It is a bar, a 3-dimensional object, so we need to consider the parts that are not in the galactic plane. These parts could move at a really high speed toward or against us, creating huge Doppler shift.

Another more likely explanation for this difference is that in the direction of longitude of 0 , there are two parts of signals that fill our sight. One is the signal from the near-center part of our galaxy, which does move with a great speed in the observation direction relative to us. This part contains low signal intensity, since only the near-center part, which is actually a point, has such a huge velocity. Another is the signal that lies on the observation line but is not emitted at the place near to the center of our galaxy. This part contains most of the signal power, since most substance-nucleus and particle that release $21 \mathrm{~cm}$ line have little Doppler speed in our observation direction. However, this also make it unclear to determine the border, since most parts of the substance - at different speeds and in different directions - are being observed.

Here, we have used some advanced methods like Gaussian functions and FWHM measuring, via an 18-meter radio telescope, to finish the thickness measurement. Some tips taken during the process greatly declined our inaccuracy, even though problems still remain. Future studies, I believe, will use more accurate methods and/or theories, including the density as a function of galactic latitude, to achieve more accurate results.

\section{Acknowledgement}

I want to thank Prof Daniel Marlow especially, for he had provided me data and helped me with coding in Python. He had also given me lectures on fundamental radio astronomy from which I learned a lot.

\section{Conflict of interests}

The authors declare that there is no conflict of interests regarding the publication of this paper. 


\section{References}

Assis, A. K. T., \& Neves, M. C. D. (1995). History of the 2.7 K Temperature Prior to Penzias and Wilson (PDF). Apeiron, 2(3).

Born, M., \& Wolf, E. (1999). Principles of Optics (p. 461). Cambridge University Press. ISBN 0-521-64222-1

Bradley, W., \& Carroll, D. A. O. (2017). Modern Astrophysics Cambirdge Universty Press. ISBN978-1-10842216-1

Sher, D. (1968). The Relativistic Doppler Effect. Journal of the Royal Astronomical Society of Canada, 62, 105111.

\section{Copyrights}

Copyright for this article is retained by the author(s), with first publication rights granted to the journal.

This is an open-access article distributed under the terms and conditions of the Creative Commons Attribution license (http://creativecommons.org/licenses/by/4.0/). 